\title{
La Corte Penal Internacional: abriendo caminos
}

Este comentario, junto a los documentos que analiza, está disponible en www.anuariocdh.uchile.cl
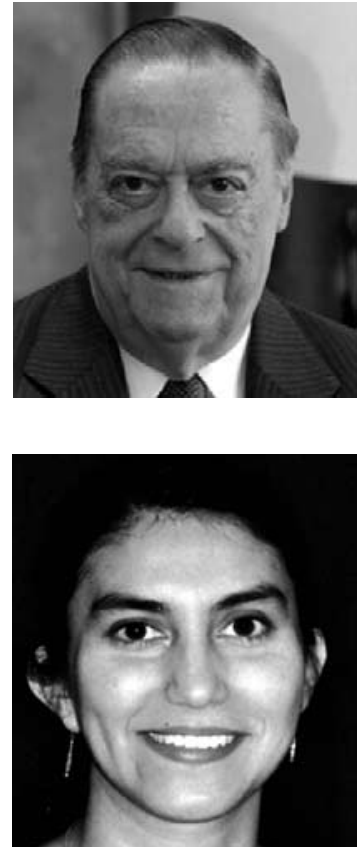

\section{Alfredo Etcheberry}

Licenciado en Ciencias Jurídicas y Sociales de la Universidad de Chile y Master en Derecho Comparado por la Universidad de Columbia, EE.UU. Profesor Titular del Departamento de Ciencias Penales de la Facultad, imparte la cátedra de Derecho Penal Internacional. Fue Vicerrector Académico de la Pontificia Universidad Católica de Chile. Entre sus principales obras están "Concurso Aparente de Leyes Penales", "American-Chilean Private International Law", "Derecho Penal en la Jurisprudencia" y "Derecho Penal", además de diversos artículos sobre Derecho Penal. alfredo@etcheberry.com

\section{Claudia Cárdenas}

Licenciada en Ciencias Jurídicas y Sociales de la Universidad de Chile, Magistra Legum (LL.M. y Doctor iuris (Dr. iur.) de la Universidad Humboldt (Berlín), autora de varias publicaciones en derecho penal internacional especialmente referidas a la Corte Penal Internacional. Departamento de Ciencias Penales, Facultad de Derecho, Universidad de Chile.

ccardenas@derecho.uchile.cl

\section{RESUMEN}

A partir del análisis de las decisiones de la Corte Penal Internacional en el Caso Lubanga y la solicitud del Fiscal de detener al Presidente en ejercicio de Sudán, los autores analizan los desafíos y perspectivas del trabajo de la Corte Penal Internacional, particularmente en lo relativo a cómo se ha configurado su labor después de una etapa inicial dedicada a esclarecer su rol y constitución.

\section{Introducción}

Si bien la Corte Penal Internacional continúa siendo una institución relativamente nueva en el concierto internacional, puede sostenerse que ya ha dejado una fase primordialmente organizativa que la ocupó durante sus primeros años para entrar de lleno en operación. Esta operación implica, conforme a su mandato ${ }^{1}$, la investigación y enjuiciamiento de los más graves crímenes

\footnotetext{
Según se desprende del Estatuto de Roma de la Corte Penal Internacional (en la versión corregida de 10 de noviembre de 1998, de 12 de julio de 1999, de 30 de noviembre de 1999, de 8 de mayo de 2000, de 17 de enero de 2001 y de 16 de enero de 2002), A/CONF.183/10, 17 de julio de 1998 (en adelante: ECPI).
} 
de derecho internacional que se encuentren dentro de su competencia material, vale decir, por ahora, el genocidio ${ }^{2}$, los crímenes de lesa humanidad ${ }^{3}$ y los crímenes de guerra ${ }^{4}$. Conforme al artículo 5 del ECPI, la Corte también tiene competencia respecto del crimen de agresión. Sin embargo, su competencia en este punto está suspendida hasta que se logre una descripción de la conducta punible ${ }^{5}$. El ejercicio de esta jurisdicción está regulado conforme al principio de complementariedad, de modo que la Corte solamente está facultada para investigar y conocer de los casos más graves que no hayan sido ni estén siendo investigados ni juzgados por otra jurisdicción de manera seria, lo que será revisado por la sala de cuestiones preliminares antes de autorizar el inicio de un procedimiento ${ }^{6}$.

Aunque en la última década se ha tornado corriente que se sigan juicios por crímenes de derecho internacional ante tribunales internacionales - gracias al trabajo de los Tribunales Penales Internacionales ad hoc para la ex Yugoslavia y para Ruanda, establecidos en 1993 y 1994 respectivamente al alero del Consejo de Seguridad de Naciones Unidas- lo cierto es que, por ser la Corte Penal Internacional el primer tribunal internacional con competencia penal de carácter permanente y sobre todo porque el principio de complementariedad en el que basa su actuar, da a su regulación una mayor complejidad que la existente en los demás tribunales en ejercicio, por lo que su actuar ha sido necesariamente pionero en varios aspectos.

Ya que esta colaboración se centra en el acontecer del año 2008, además de una breve reseña general sobre el funcionamiento de la Corte en ese periodo, destacaremos dos asuntos que muestran de manera particularmente elocuente el modo en que la Corte está llevando a cabo su mandato: (i) la decisión que estuvo por significar la liberación del primer detenido ante la Corte, (ii) y la solicitud de la Fiscalía de dictar una orden de detención contra el presidente en ejercicio de Sudán Omar Hassan Ahmad Al Bashir. Optamos por esta forma de proceder con la convicción de que examinando estos dos hitos se podrán comprender de mejor forma las fortalezas o desarrollos que implican avances en el actuar de la Corte y debilidades o desarrollos que se pueden calificar de cuestionables, y cuya superación representa desafíos para la justicia penal internacional.

\section{El funcionamiento de la Corte: fortalezas y debilidades}

Como en toda institución, particularmente cuando se asume el ejercicio de verla desde fuera, pueden observarse fortalezas o desarrollos que implican avances y debilidades o desarrollos que pueden calificarse de cuestionables, y cuya superación representa desafíos.

Dentro de las fortalezas, están aquellas que ya poco nos sorprenden respecto del año anterior: el alto grado de compromiso de su personal; el gran número de Estados Partes (108), lo que le da un buen soporte de legitimidad; el hecho de que varios procedimientos al interior de la Corte se han ido estandarizando, esclareciéndose competencias específicas en la organización, por

2 Conforme al artículo $6 \mathrm{ECPI}$, que castiga ciertos actos perpetrados con la intención de destruir total o parcialmente a un grupo nacional, étnico, racial o religioso como tal.

3 Conforme al artículo 7 ECPI, que castiga ciertas conductas llevadas a cabo como parte de un ataque generalizado o sistemático contra una población civil y con conocimiento de dicho ataque.

4 Conforme al artículo $8 \mathrm{ECPI}$, que distingue entre crímenes de guerra en conflictos armados internacionales y crímenes de guerra en conflictos armados no internacionales.

5 Su tipo penal aún debe ser definido por una modificación del Estatuto (artículos 5, 121 y 123 ECPI). Conforme al artículo 121 inciso 1 ECPI las modificaciones al Estatuto sólo podrán tener lugar a partir del séptimo año de entrada en vigor del tratado -esto es, a partir del 1 de julio de 2002- y requieren de una mayoría de siete octavos de sus Estados Partes (artículo 121 inciso 4).

6 Sobre las limitaciones jurídicas al ejercicio de jurisdicción por parte de la Corte, cfr. en particular los artículos 1, 11, $12,17-20,53 \mathrm{ECPI}$. 
ejemplo, cuando se desea cursar una solicitud de cooperación, y la transparencia -al parecer no es en vano que su Fiscal Luis Moreno Ocampo fuera Presidente de Transparency International para América Latina y el Caribe. Las audiencias pueden seguirse en video, pueden revisarse sus actas, se encuentran copias de todas las resoluciones en cada caso, en fin, muchísima información es accesible en Internet de manera expedita. Se sigue en esto la senda que ya han marcado los tribunales penales internacionales ad hoc. De este modo, el actuar de la Corte está permanentemente sometido al escrutinio público, lo que a largo plazo resulta sin duda provechoso.

Ya en lo contingente del año 2008, también el hecho de que el Fiscal haya solicitado la detención de un jefe de Estado en ejercicio (en lo que se ahondará infra) significa -más allá de los juicios que el caso concreto merezca- que se procura investigar y someter a juicio a quienes según la información disponible corresponda, más allá de su cargo oficial. Si bien esta posibilidad está explícitamente abierta conforme al art. 27 párr. 1. ECPI, la Fiscalía ha demostrado que está dispuesta a utilizar esta atribución sin tomar el camino de procurar evitarse problemas, sino que está dispuesta a enfrentarlos. También ha de citarse como fortaleza la decisión de hacer prevalecer los derechos del imputado de estos horrendos crímenes por sobre otras consideraciones también atendibles. Así quedó de manifiesto en el caso Lubanga, que se comentará más adelante, y que echa por tierra con hechos las alegaciones de quienes sostienen que la Corte busca condenas a cualquier precio.

Entre las debilidades de la Corte como institución puede mencionarse que los funcionarios de la Corte están bastante sobrecargados de trabajo con las cuatro situaciones que están siendo objeto de investigación (Uganda, República Democrática del Congo, Sudán-Darfur y República Centroafricana), lo que hace que no puedan dedicarse a nuevas investigaciones que se harían igualmente necesarias. Si bien al revisar el trasfondo de cada situación no puede desconocerse que cumplen con creces con todos los parámetros de gravedad imaginables, el hecho de que todas estas situaciones tengan lugar en África y de que no se vislumbre la posibilidad a corto plazo de abrir nuevas investigaciones hace que se imponga la crítica de que la Corte se ocupa sólo de juzgar a africanos, o en todo caso a ciudadanos de Estados sin gran poder en el concierto de potencias mundiales. Esto podrá ser así. Pero por otra parte, precisamente el olvido del que por décadas han sido objeto estas regiones para gran parte del globo, ha permitido que tengan allí lugar crímenes de las características y en la escala que lamentablemente han existido, y el hecho de que por fin se pretenda investigar y sancionar seriamente no puede ser cuestionado. Claro está que lo óptimo sería contar con más recursos, pero hay que juzgar la labor que se hace conforme a los recursos de los que efectivamente dispone la institución.

Desde el punto de vista político, suele citarse como debilidad el hecho de que la Corte aún no cuente con la ratificación de importantes potencias mundiales (de los miembros permanentes del Consejo de Seguridad, sólo son partes el Reino Unido y Francia). Sin embargo, no puede desconocerse que la institución sí cuenta con un amplio respaldo de los Estados democráticos, a pesar de la activa campaña de Estados Unidos por evitarlo.

\section{Los hitos del año 2008}

\section{a. Decisiones en el Caso Lubanga}

Las investigaciones en los casos de competencia de la Corte son por naturaleza complejas, no solamente en lo jurídico, sino también desde lo logístico-práctico, pues se actúa en zonas de conflicto, debiendo obtenerse la cooperación de Estados u organizaciones en terreno para poder operar. Luego, resulta altamente difícil obtener la detención de una persona y es todo un hito que se logre llevar a un imputado a juicio. Esto, que es así en todos los casos, fue particularmente 
cierto en el caso del primer imputado que se presentó ante la Corte, el primer detenido ante la Corte Penal Internacional, Thomas Lubanga Dyilo. Thomas Lubanga fue detenido en Kinshasa (Capital de la República Democrática del Congo) el 17 de marzo de 2006 y fue entregado a la Corte. Es de nacionalidad congolesa y sería fundador y líder de la Union des Patriotes Congolais (UPC). Se lo acusa de crímenes de guerra, en particular de reclutar o alistar a niños soldados (menores de 15 años) o utilizarlos para participar activamente en las hostilidades ${ }^{7}$. La detención se obtuvo con la cooperación de las autoridades congolesas, que fueron quienes antes habían remitido el caso a la Corte $^{8}$.

Con los ojos del mundo expectantes, el proceso iba avanzando. Entretanto había otros acusados ante la Corte, pero este sería el primer procedimiento en llegar a la etapa de juicio. Con todo, el inicio del juicio quedó en suspenso cuando, el 13 de junio de 2008, la Sala I de Primera Instancia ${ }^{9}$ decretó el aplazamiento de las actuaciones del procedimiento ${ }^{10}$, ya que la Fiscalía no podía desclasificar documentos que había obtenido mediante convenios a los que la faculta el art. 54 párr. 3 e) $\mathrm{ECPI}^{11}$, mientras la defensa alegaba su derecho de conocerlos para poder defender adecuadamente al imputado.

Si bien para quienes estaban al tanto de los términos del Estatuto y del acuerdo de cooperación, por ejemplo, entre la Organización de las Naciones Unidas y la Corte Penal Internacional ${ }^{12}$, el fundamento del problema no fue demasiado sorpresivo, había de todas formas expectación por saber la forma en que la Corte resolvería este conflicto: la Corte debía ponderar, por una parte, el derecho a una adecuada defensa del imputado, y por otra, la facultad del Fiscal para garantizar confidencialidad como único modo posible de obtener cooperación en ciertos casos. Aunque se esperaba que la Sala de Primera Instancia debía hacer "algo" frente al dilema que se le presentaba, para muchos pareció demasiado abrupto que ese "algo" fuera que, el 2 de julio de 2008, la misma Sala de Primera Instancia resolviera dejar en libertad al inculpado Thomas Lubanga Dyilo, por considerar que no estaban dadas las garantías de un debido proceso. Dicha resolución no debía ejecutarse sino cinco días después, tiempo en que las partes podían apelar de ella.

Esta decisión causó airadas reacciones, pues aunque por una parte existía consenso en la necesidad de buscar una fórmula en que el acusado pudiese ejercer plenamente sus derechos, la resolución de 2 de julio, de llegar finalmente a ejecutarse, podía significar echar por la borda años de trabajos investigativos, por una parte; por otra, al menos para un sector de la opinión pública, la impresión de que la Corte no estaba cumpliendo su misión -la conocida alegación de la puerta giratoria, ahora a nivel internacional- ya que a las víctimas se les habría dado una esperanza para ahora volver a abandonarlas. Hubo quien incluso solicitó la renuncia inmediata

7 Más información en >http://www.icc-cpi.int/Menus/ICC/Situations+and+Cases/Situations/Situation+ICC+0104/ Related+Cases/ICC+0104+0106/Democratic+Republic+of+the+Congo.htm<, visitado el 28 de febrero de 2009.

8 Si bien la Corte solo conocerá de casos que hayan tenido lugar después del primero de julio del 2002, ya desde la década de los 90 varios miles de civiles han muerto como resultado del conflicto en la República Democrática del Congo. Entre las conductas delictivas más recurrentes se cuentan las ejecuciones masivas, violaciones, torturas, desplazamiento forzoso de la población y utilización de niños soldados.

9 Integrada por los jueces Fulford (Presidente), Blattmann y Odio Benito.

10 Cfr: > http://www.icc-cpi.int/iccdocs/doc/doc511249.PDF<, visitado el 1 de marzo de 2009.

11 Según esta norma, el Fiscal podrá convenir que no divulgará en ninguna etapa del procedimiento los documentos o la información que obtenga a condición de preservar su carácter confidencial y únicamente a los efectos de obtener nuevas pruebas, salvo con el acuerdo de quien haya facilitado la información.

12 http://www.icc-cpi.int/NR/rdonlyres/3EBEECA5-0EAC-4685-950F-1488CF56BE64/0/ICCASP3Res1_Spanish.pdf 
del Fiscal ${ }^{13}$. Quienes habían cooperado con la Corte temieron por su seguridad en caso de que Lubanga fuera liberado, pues esto podría exacerbar el conflicto en Ituri ${ }^{14}$.

Desde otro punto de vista, la resolución fue interpretada como una muestra de independencia y probidad (fairness) de los jueces, cuestión fundamental para que la Corte siga teniendo apoyo de la comunidad internacional. Hubo entonces quienes optaron por criticar no a la Corte, sino a las fuentes que no permitían la desclasificación de las pruebas para la Defensa, sobre todo en el caso de las pruebas obtenidas a través de la cooperación con las Naciones Unidas ${ }^{15}$.

Lo anterior, porque si las evidencias se han obtenido bajo reserva de confidencialidad, sólo pueden desclasificarse previo acuerdo con la fuente. En este caso estaban en juego más de 100 piezas de documentación obtenidas con la colaboración de Naciones Unidas, que a través de una carta fechada el 20 de junio de 2008 sólo autorizaba a los jueces a revisar los documentos, en dependencias de Naciones Unidas, sin posibilidad de sacar copias o tomar notas, entre otras restricciones.

Como era de esperar, las partes apelaron. Desde el punto de vista jurídico ${ }^{16}$, son principalmente dos las disposiciones en conflicto. Por una parte, el artículo 54 párrafo 3 e) ECPI faculta al Fiscal para llegar a acuerdos para mantener reserva durante todo el procedimiento de documentos o información obtenidos bajo reserva de confidencialidad, y sólo con el propósito de obtener nueva evidencia. Dicha confidencialidad sólo puede ser alzada si el proveedor de la información consiente en ello. Por otra, el artículo 67 párrafo $2 \mathrm{ECPI}$ señala que el Fiscal deberá, tan pronto como le sea posible, desclasificar para la defensa toda evidencia exculpatoria que se encuentre a su disposición, así como la que pueda contener información que sirva para alegar una mitigación de la responsabilidad penal o pueda afectar la credibilidad de la evidencia de la Fiscalía.

La Sala de Apelaciones tardó en resolver. El asunto se solucionó, en gran medida, porque en los días siguientes la Organización de Naciones Unidas flexibilizó su posición en cuanto a la confidencialidad de los documentos en cuestión, facilitando su accesibilidad para los jueces e incluso por su intermedio para la defensa ${ }^{17}$.

El 21 de octubre de 2008 la Sala I de Apelaciones ${ }^{18}$ dictó dos resoluciones: en la primera se deniega la solicitud del Fiscal de dar curso inmediato al juicio oral ${ }^{19}$, mientras que en la segunda, referida a la liberación de Lubanga, la Sala de Apelaciones resolvió reenviar el asunto a la Sala de Primera Instancia de modo que lo revisara habida cuenta de los antecedentes actuales ${ }^{20}$.

Actualmente el proceso sigue su curso y la primera audiencia de éste -el primer juicio ante la Corte Penal Internacional- tuvo lugar el 26 de enero pasado, dándose así cumplimiento a la decisión

13 Cfr. Joshua Rozenberg (Telegraph-UK), 2 July 2008.

14 http://www.redress.org/news/08-07-03\%20Lubanga\%20ICC\%20-\%20REDRESS\%20Press\%20Release\%20-\%20FINAL.pdf

15 Cfr. > http://www.dia-afrique.org/suite.php?newsid=6445<, visitado el 1 de marzo de 2009: "La Coalition nationale pour la Cour pénale internationale $(C N-C P I)$ en République Démocratique du Congo, $R D C$, invite l'Onu à démontrer sa volonté de promouvoir la paix et la sécurité en coopérant pleinement avec la justice internationale dans le dossier Thomas Lubanga Dyilo."

16 Cfr. también, sobre el punto, el texto de Eugene Bakama Bope (AR No. 181, 08-Aug-08) en >http://www.iwpr. net/?p=acr\&s=f\&o=346057\&apc_state=henh<, visitado el 1 de marzo de 2009.

17 En la práctica, los jueces pueden obtener copia de los documentos y, de ser necesario, realizar resúmenes para la defensa, según publicara el 11 de julio 2008 >http://www.usatoday.com/news/world/2008-07-11-International-Criminal-Courttrial_N.htm<, visitado el 1 de marzo de 2009.

18 Integrada por los jueces Song (presidente), Kirsch, Pikis, Kourula y Ntanda Nsereko.

19 En español en >http://www.icc-cpi.int/iccdocs/doc/doc629501.pdf<, visitado el 1 de marzo de 2009.

20 En español en >http://www.icc-cpi.int/iccdocs/doc/doc627232.pdf<, visitado el 1 de marzo de 2009. 
del 18 de noviembre del año pasado que indicó que las razones que habían llevado a resolver la suspensión del proceso contra Lubanga ya no existían ${ }^{21}$.

En definitiva, se buscó arduamente, y se encontró, una vía de solución que desentrampa una materia de vital importancia para todos los procesos futuros, ya que para todos ellos será difícil y necesario reunir pruebas y en todos ellos ha de respetarse el derecho de defensa del imputado.

\section{b. La solicitud de detención de un jefe de Estado en ejercicio}

Paralelamente al desarrollo recién esbozado, otra situación ante la Corte Penal Internacional que ha dado también bastante de que hablar ha sido la situación Sudán-Darfur, que fuera remitida a la Corte por el Consejo de Seguridad de Naciones Unidas mediante la resolución 1593 (2005). La controversia surgió luego de que el 14 de julio de 2008 el Fiscal de la Corte Penal Internacional, Luis Moreno Ocampo, solicitó a la Sala de Cuestiones Preliminares que emitiera una orden de detención en contra del Presidente en ejercicio de Sudán, Hassan Ahmad Al Bashir, por genocidio, crímenes de lesa humanidad y crímenes de guerra en Darfur ${ }^{22}$.

Con su solicitud, la Fiscalía presentó a la Sala de Cuestiones Preliminares evidencia de que Al Bashir habría ideado e implementado un plan para destruir sustancialmente a los grupos étnicos de Fur, Masalit y Zaghawa. Estos grupos, históricamente influyentes y ahora marginados en la provincia, se habrían rebelado, dando a Al Bashir la excusa perfecta para combatirlos como a grupos de insurgentes.

Según la tesis de la Fiscalía, sería el Presidente quien estaría al mando de las milicias Janjaweed, que tanta desolación han causado en Darfur, destruyendo numerosos poblados, matando personas, llevando reiteradamente a cabo violaciones en grupo. Por más de cinco años el Presidente de Sudán ha negado los crímenes, Ilegando a sostener que la violación no existe en Sudán. Esta conducta del Presidente, siguiendo a la Fiscalía, ha hecho posible la comisión de nuevos crímenes.

Desde el inicio del trabajo de la Corte Penal Internacional, la Fiscalía no había solicitado la detención de un Presidente en ejercicio. Como era esperable, la solicitud de Moreno Ocampo despertó controversia: por una parte, sobre todo los Estados árabes señalan que pone en peligro el proceso de paz de Sudán y, por otra, se cuestiona que exista siquiera una etapa de transición hacia la paz en Sudán, por lo que ésta, por ser inexistente, no podría correr peligro.

Siria $^{23}$, cuyo Presidente era a la sazón también Presidente de la Liga Árabe, manifestó su oposición a una posible detención, considerándola una flagrante intromisión en sus asuntos internos. También se manifestaron en contra autoridades de Argelia, Irán, Eritrea, Jordania, Tanzania y Yemen $^{24}$. Se apresuraron a decir que una decisión en ese sentido pondría en peligro el proceso

21 Esto lo hace el Fiscal acompañando evidencia que haga que parezca razonable creer que la persona tiene responsabilidad penal por los crímenes que se le imputan. Cfr. >http://www.icc-cpi.int/menus/icc/press \%20and\%20media/press \%20 releases/press \%20releases $\% 20(2008) /$ stay $\% 20$ of $\% 20$ proceedings $\% 20$ in $\% 20$ the $\% 20 l u b a n g a \% 20$ case $\% 20$ is $\% 20$ lifted $\% 20$ _\%20trial\%20provisionally\%20scheduled\%20for\%2026\%20january\%20200<, visitado el 1 de marzo de 2009.

22 Cfr. > http://www2.icc-cpi.int/menus/icc/situations\%20and\%20cases/situations/situation\%20icc\%200205/press\%20 releases/a <, visitado el 1 de marzo de 2009.

23 Cfr. >http://www.alalam. ir/english/ en-NewsPage. asp?newsid=0310301200807222 00020<, visitado el 1 de marzo de 2009.

24 Cfr. >ttp://www.iranmani a.com/News/ ArticleView/ Default.asp? NewsCode $=60157 \&$ NewsKind $=$ Current $\% 20$ Affairs $<$, > http://allafrica.com/stories/200807210101.html<, > http://allafrica.com/stories/200807221172.html<, > http://www. sabanews.net/en/news159351.htm<, visitados el 1 de marzo de 2009. 
de paz en Sudán ${ }^{25}$, que obedecería sólo a razones políticas y sería ilegal. Algo más tarde, la Unión Africana hizo un Ilamado al Consejo de Seguridad para que hiciese uso de la atribución que le otorga el artículo 16 ECPI, de suspender la investigación por una resolución ${ }^{26}$, lo que en definitiva no ha ocurrido ${ }^{27}$.

Sin embargo, también ha habido reacciones positivas ante la solicitud. Hay quienes sostienen que con esto se le da credibilidad a la Corte $^{28}$, al procurar perseguir efectivamente a los responsables de más alto rango.

Siendo la Corte Penal Internacional un tribunal internacional permanente y no uno establecido con el solo propósito de investigar una situación particular, es necesario establecer mecanismos para decidir qué casos va a conocer y cuáles no. En materia de negociaciones de paz en curso, la declarada política del Fiscal es no intervenir en casos donde el proceso de paz pueda verse puesto en peligro. En lugar de ello, la Fiscalía preferirá esperar, estando expectante al posterior desenvolvimiento de los hechos. Esta política es altamente relevante, ya que la Corte Penal Internacional es Ilamada precisamente a ejercer su jurisdicción en situaciones de conflicto. Ciertamente es posible que en ocasiones las partes de una negociación de paz puedan amenazar con abortar la negociación si la Corte Penal Internacional inicia investigaciones en la situación involucrada, pero existen en el Estatuto mecanismos de resguardo para estos $\operatorname{casos}^{29}$. Por ello, un análisis acucioso de la situación en Darfur excede la finalidad de estas páginas, sin embargo, para poder tomar partido en un sentido o en otro es necesario dilucidar si existe realmente un proceso de paz en curso que pudiera eventualmente verse dañado por un proceso.

La salvedad queda hecha puesto que, sin perjuicio de lo recién expuesto y precisamente por ello, hace falta poner atención en que el mero invocar un peligro para negociaciones de paz sirva como un pretexto para bloquear el actuar de la Corte, debilita la gran ventaja que representa el carácter permanente de la Corte Penal Internacional frente a los tribunales penales internacionales que le han precedido, haciendo demasiado fácil para autores de crímenes internacionales el evitar la persecución internacional alegando que los procedimientos irían en contra del interés de la justicia o de la paz, mientras se esmeran por mantener vivo el fantasma de la inestabilidad al menos hasta que llegue el tiempo en que ya sea muy tarde para una investigación y persecución penal exitosas. De allí que sea de primordial relevancia el determinar en qué casos efectivamente existe un proceso de paz en curso que pueda ser dañado.

En el caso de Darfur, el 5 de mayo de 2006, en Abuja, el gobierno de Sudán y el Sudan Liberation Movement/Army (conocida como fracción Minni Minawi) firmaron el acuerdo de paz de Darfur. ¿Basta eso para que se considere que hay un proceso de paz en curso que se pudiera poner en peligro? La respuesta es categóricamente negativa, porque a casi tres años de ello la situación sigue siendo altamente conflictiva, habiéndose vencido holgadamente los plazos fijados en el acuerdo (artículo 30) para cumplir las diversas etapas allí previstas.

25 Cabe señalar que poco después de conocida la noticia, el presidente viajó a Sudán a prometer paz, Cfr. >http://www. sudantribune.com/spip.php?article27954<, > http://afp.google.com/article/ALeqM5irq8g_TRaIRaUDO9WFiXZbNSItU $\mathrm{w}<$ visitados el 1 de marzo de 2009.

26 Cfr. > http://afp.google.com/article/ALeqM5j_oyQDTMFTjR2o7kNThR_10pEITg<, > http://english.aljazeera.net/news/africa /2008/07/200872114839853387.html <, >http://www.monitor.co.ug/artman/publish/news/AU_seeks_to_delay_Al-Bashir_ indictment_68665.shtml<, >http://www.sudantribune.com/spip.php?article28156<, visitados el 1 de marzo de 2009.

27 Si bien el embajador de Rusia ante Naciones Unidas declaró que podrían considerarlo, cfr. >http://news.xinhuanet.com/ english/2008-07/22/content_8744731.htm<, visitado el 1 de marzo de 2008.

28 Cfr., por todos, el artículo de David Rohde en >http://www.nytimes.com/2008/07/22/world/europe/22tribunal.html?_r=2<, visitado el 1 de marzo de 2009.

29 Cfr. principalmente los arts. 16 y 53 ECPI. 
En reiteradas ocasiones, el Presidente Al Bashir ha desconocido la jurisdicción de la Corte ${ }^{30}$ señalando que el poder judicial de Sudán está calificado para juzgar a cualquier sudanés, por lo que no se entregará a ningún ciudadano sudanés para que sea juzgado en el exterior. Este discurso, escuchado superficialmente, puede generar simpatías. En efecto, si se juzgaran los crímenes cometidos en Sudán en el propio Sudán, ciertamente la Corte no estaría facultada para proceder. El punto es que a la fecha no se ve que ese Estado se esté ocupando de la serie de gravísimos delitos que se cometen en la región de Darfur, y que han motivado, entre otros fenómenos, el desplazamiento de la población y una crisis de refugiados en los vecinos países vecinos de Chad y la República Centroafricana, desestabilizando a toda la región en lo que viene siendo desde hace años una verdadera tragedia para la humanidad, por las condiciones subhumanas que allí imperan.

La Sala de Cuestiones Preliminares aprobó la solicitud del Fiscal y fue emitida la orden de detención solicitada contra Al Bashir, si bien se excluyó de ella el delito de genocidio. Como no es de extrañar, la reacción de Al Bashir fue airada en términos destemplados; el afectado señaló que no tenía intenciones de cumplir con lo ordenado por la Corte, y en los días siguientes procedió a expulsar de Sudán a diversos organismos principalmente de carácter humanitario, medida que no fue formalmente vinculada a la orden de detención contra el Presidente, pero cuya relación es indudable. Los acontecimientos son todavía muy recientes para vaticinar su evolución. Como se ha dicho, es previsible que en el futuro se produzcan casos en que haya cierta tensión entre la necesidad de paz y seguridad, función primordial de Naciones Unidas, y la investigación y enjuiciamiento de gravísimos delitos internacionales, que es la función de la CPI.

\section{A modo de conclusión}

El carácter inédito de un tribunal internacional con carácter permanente hace que inevitablemente en muchos aspectos la Corte Penal Internacional deba necesariamente transitar por terra incognita. De allí que sea natural que haya opiniones que apoyen las decisiones de la Corte y otras que no lo hagan. El gran interés que despierta el actuar de la Corte a nivel internacional hace que se vea en la obligación de fundamentar adecuadamente cada decisión. Durante el 2008, de sus actuaciones pueden desprenderse principalmente dos mensajes. Por parte de los jueces, que éstos actúan en forma independiente de la Fiscalía, sirviendo de efectivo control de sus actuaciones en resguardo de las garantías jurídicas. Por parte de la Fiscalía, que ésta no se conforma con lo que pueda obtener mediante el camino más fácil, sino que está en condiciones de enfrentar las dificultades que haga falta en pos de cumplir adecuadamente con su mandato de investigar, acusar y seguir un juicio con las debidas garantías en contra de quienes aparezcan como responsables de los más graves crímenes de trascendencia internacional. Más allá de los detalles de cada caso, la síntesis de ambos mensajes hace augurar un futuro complejo, pero esperanzador.

$30 \mathrm{Y}$ junto con ello se ha negado a cooperar, como debiese hacerlo en virtud de la resolución del Consejo de Seguridad 1593 (2005) que remite la situación a la Corte. 